\title{
Design and Development of Virtual Learning Environment Using Open Source Virtual World Technology
}

\author{
Steven Suman ${ }^{1}$, Ardavan Amini ${ }^{1}$, Bruce Elson ${ }^{1}$, and Patricia Reynolds ${ }^{2}$ \\ ${ }^{1}$ Birmingham City University, UK \\ ${ }^{2}$ King's College London - UK \\ suman.steven@googlemail.com, ardavan.amini@tic.ac.uk, \\ bruce.elson@tic.ac.uk, p.a.reynolds@kcl.ac.uk
}

\begin{abstract}
Today's digital era, dynamic teaching and learning in higher education has moved from traditional class room, face-to face learning environments to more interactive and collaborative environments, due to a demand for onlinedistance learning from students, and the desire from academic institutes to promote and deliver courses across the globe. Advancement in open source virtual learning platforms technology, enables the development of flexible online learning environments to exist that can be accessed anywhere, anytime and in anyplace by students, hence enabling academics and academic institutes to teach and increase their market across the globe. Authors of this paper had the opportunity to work on the design and development of a flexible virtual learning environment within the dentistry sector part of the IVIDENT (virtual dental school) UK Higher Education Funding Council project at Kings College London. This paper will focus on the design and the development of virtual learning environment for the IVIDENT project, using advanced technological open source virtual world platforms.
\end{abstract}

Keywords: Project wonderland, architecture, 3d modelling, haptics, java technology, open source technologies.

\section{Introduction}

In the recent years technology has seen the development and popular use of virtual online worlds [1], early stages of online virtual world primary uses were social based, and a meeting point for end users around the world to interact with one another. As online virtual worlds have developed, many organisations and industries not just associated with information systems are turning to virtual online worlds to host virtual presentations and meeting's, with employees and partners interacting from mobile locations around the world on smart clients as well as desktop computers. The uses of virtual worlds provide many benefits, reduced costs of training staff via simulations and rich online media.

Due to the increasing demand from the educational establishment to enhance their delivery of learning material to be more flexible, accessible and available, The UK \& 
European Union is funding research projects in this particular area, for development of online virtual learning environments. The IVIDENT project based at Kings College London is a Higher Education Funding Council UK project for development of an online virtual learning platform for the training of dentists.

The research into virtual world technologies available on the market resulted in identifying a key common issue, that of virtual worlds availability and uses primarily for social networking and gaming. Recent uses of virtual worlds have resulted in an educational nature, but are yet to be dominant and mainstream as an acknowledged additional resource to aid in delivering effective learning within academic institutions.

The authors of this paper have designed and developed a prototype virtual learning environment, using the open source virtual world development kit Project Wonderland, as enhancement to the IVIDENT UK project.

\section{Need for Change}

Traditional methods of teaching within class rooms, lectures and face to face interaction, increases costs within the educational system, limits academic institutions from offering additional further education courses, creates unavailability of staff for teaching additional courses and limits distant learning. A paper written in 2005 by Marileena Koskela titled 'Suitability of a Virtual Learning Environment for Higher Education,' at Tampere University in Finland, conducted tests to determine how effective traditional teaching methods are compared to using e-learning resources within a virtual learning environment.

Tests included creating groups for both students and lecturers and delivered the course material to both groups, one group would interact with virtual learning environment and the other traditional education methods.

Student's performances were analysed and students using virtual learning environments preceded that of traditional methods and also found the course understanding easier to digest [2], for selected courses other tests conducted identified using virtual learning environment made little or no difference at all, also virtual learning environments proved to be a success within higher education.

The limitaitons with traditional methods is that of students embarassment of asking questions on the course material due to lack of knowledge and understanding, a key problem in both class room and lecture teaching, and many students also don't participate and fall asleep within lectures. This is a common problem across many further education academic institutions. Using a virtual learning environemt the experience becomes personal for the user, and constant interaction and engagement with the user is required. Students who are embarassed to ask questions are able to follow virtual course material at a suitable pace, and interactive applications can be developed to provide a futher detailed understanding of the material. Teaching staff are able to address a larger cohorts of students to deliver course material delivered more than once.

Students studying dental education lack the real world dentistry experience and currently work with laboratory dummies to conduct dental procedures, fillings, crowns and many more. The key element for students obtaining the real world experience is creating an immersive environment via utilising three dimensional 
graphics technologies to simulate realistic dental procedures and objects and with the use of haptic devices will aid in students touch and feels senses and provides a valuable resource.

\section{Methodology}

There are many system development life cycles, Structured Analysis and design (SSADM), Rapid Application Development (RAD) and the Spiral Model. Within the time frame feasible to develop a prototype, the Spiral Model was adopted. The Spiral model life cycle was defined by Barry Boehm in his 1988 article A Spiral Model of Software Development and Enhancement [3].

Spiral model uses iterative development cycles via obtaining the system requirements (primary research) to develop the design of the system, implement a prototype and then begin a new iteration of analyse, design and prototype based on knowledge of previous iteration cycles until system requirements are fulfilled.

Using the spiral model has the advantage of developing a system close to the customer's requirements, because consistent communication is held between the development house and customer. Changes to the system based on new requirements can be implemented on the next iteration cycle of development.

The figure 1 illustrates the prototype project life cycle mapped onto the spiral model. The first stage begins with identifying the customer requirements, which is to develop a client application to use for the virtual dental school. Once the requirements have been identified, planning the project is the next step to identify both the scope and feasibility of the project within the time frame proposed for development of prototype software, findings from the project planning stage will also aid in identifying key objectives required to complete the project.

The project risk analysis is carried out after each-iteration of the project prototype stages, risk analysis importance is of a high priority, performing a project risk analysis will identify possible risks which may occur throughout the project life cycle and countered with solutions to areas of concern. Identifying risks at an early stage will substantially assist in the project running smoothly and meeting deadlines.

Primary and secondary research is carried out to determine further understanding of the system requirements; the process involves conducting interviews with end users and stake holders of the project, and also observing the surroundings i.e. dentist practices to create realistic models reflecting real world objects and also carry out an analysis stage, using virtual world development kits to gather results of limitations and advantages of use and cross reference the results against the system requirements and project objectives.

Development of the software phase begins immediately, with basic system design principles, because this is one of the benefits of using the spiral model for system development, prototypes are developed after basic analysis and design phases and then illustrated to the end user where new requirements can be identified or existing requirements can be changed and changes to the system will be implemented within the next iteration. The end result of the software system is close to the end users requirements. Other life cycles exist which require five phases for project development Analysis, Design, Implementation, Testing and Evaluation (SSADM), each stage 
cannot begin without the completion of the previous stage, with this methodology communication with the end user is limited and additional requirements or changes to the system are difficult to implement once into the implementation stage of the project life cycle.

The development of the three dimensional models are developed as prototypes and illustrated to stake holders of the project, at this stage the models are evaluated and if any additional requirements and modifications are noted to be implemented over the second iteration.

Further development of the system is conducted to aid in continuing with the next iteration process and also the objective are evaluated, to determine objectives have been met or altered. This process follows into the conducting another risk analysis based on the first and following second project iterations. Another prototype is developed creating or modifying existing three dimensional models and also development of the virtual dental school client, stake holders are shown the developed prototype and evaluation of the prototype is assessed, to determine further enhancements and requirements for the next iteration of the project life cycle. The project phase then enters system testing.

The project enters third development iteration, conducting the same phases from the first and second iterations, with the additional development of a system test plan.The project life cycle ends with the development of writing a detailed project report.

The spiral model was chosen as the preferred methodology to develop the virtual learning environment for the IVIDENT project. The model along with the time scales is shown in the figure 1 below

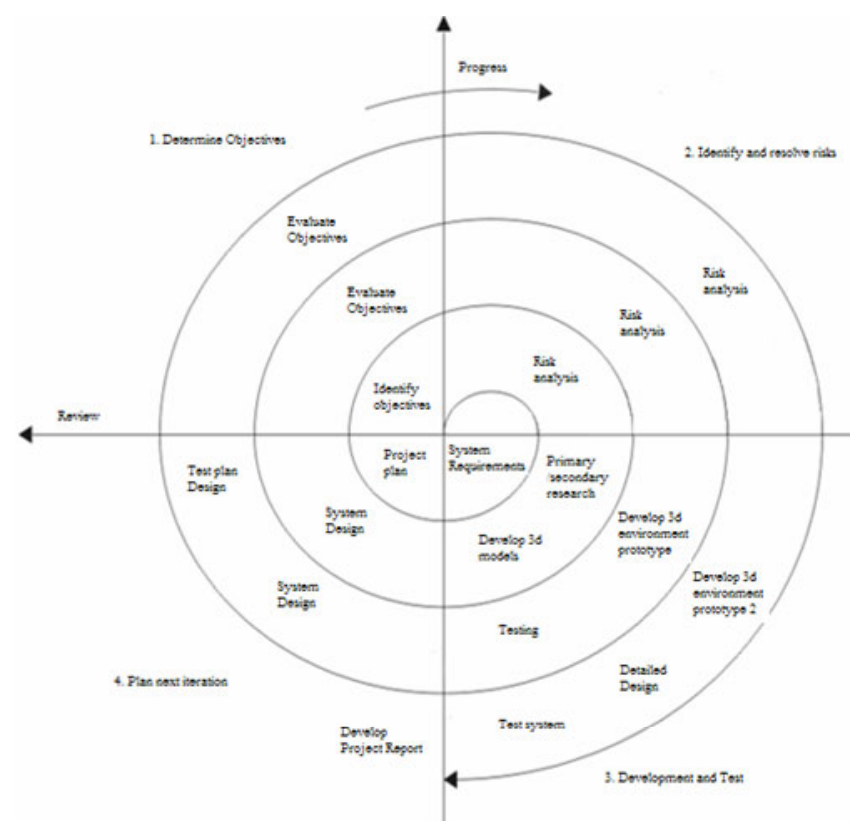

Fig. 1. Spiral Model based upon Boehm Spiral Model [3] 


\section{Virtual Worlds}

Virtual world's technology is enabling organisations to communicate and collaborate from remote locations around the globe. Online virtual worlds are decreasing costs within organisations, via virtual presentations and training simulations.

Integrating education with online virtual worlds exists to a degree. Many open universities Harvard, Texas, Stanford are developing virtual campuses to allow students to meet attend virtual classes and create content [4], but the used virtual world requires users to subscribe in order to use the services provided. Therefore open source technologies are used to develop a virtual world applicable to education and incorporate the common framework use of the educational system, to allow a generic virtual world to be developed and used within academic institutions.

\subsection{Development Kits}

Findings of open source research resulted in several virtual world development kits opensim (Microsoft), Project Wonderland (sun Microsystems) and Croquet Consortium available. Each virtual world development kit had its strengths and weaknesses based on design architecture, flexibility and ease of extending the environment further. The concept of anywhere, anytime, anyplace was not feasible with opensim because the architecture is not platform independent and additional software is required to be installed on non Microsoft window operating systems for compatibility. The scope of the croquet consortium project relies on developers developing additional functionality to the existing project, and in the long term is not applicable for IVIDENT.

As part of this project one of the main challenges was to identify an open source virtual environment that is available on the market as a software suite, which would be able to integrate with existing academic systems or academic learning tools, e.g. moodle, IVIDENT enrolment system etc. A large percentage of virtual environments researched resulted in monthly subscription payments to use the services and the host to maintain system for the end user. Other virtual worlds were hosted specifically towards virtual presentations and simulations.

\subsection{Project Wonderland}

Project Wonderland is Java an open source toolkit for creating collaborative 3D virtual worlds. Within those worlds, users can communicate with high-fidelity, immersive audio, share live desktop applications and documents and conduct real business. Wonderland is completely extendable; developers and graphic artists can extend its functionality to create entire new worlds and new features in existing worlds [5].

Sun Microsystems java project wonderland 0.4 versions were selected as the basis for developing the virtual world. Project wonderland provides a framework API library to develop virtual worlds, three dimensional models can be imported and rendered in the virtual environment in the format of X3D technologies, which can be developed within many three dimensional modelling packages available on the market. Open source modelling software blender was used as part of developing models for the virtual world. 


\subsection{D Modelling}

Three dimensional models are created from polygon meshes and are made up of triangles positioned to form the object, modelled.

Various techniques were researched into development of three dimensional modelling, and how developed models will be represented within a virtual world, without creating limitations of the hardware.

Techniques of creating low polygon meshes enable the virtual worlds to calculate and render fewer triangles which will release processing graphics information on the hardware. Increasing the polygon mesh size, results in using hardware with higher memory capacity to fulfil the graphics requests, and becomes very costly.

Back face culling is another technique used to render three dimensional models, via using graphics hardware to calculate the meshes triangle within the users view frustum.

Ian Palmer [6] explains modelling techniques for texture mapping three dimensional objects and how to preserve graphics hardware memory, which is essential to delivering high end realistic models without reaching the limitations of the hardware. The concept to texture mapping is to unwrap the mesh model into a flat blue print layout and the object vector positions are stored. The blue print layout is imported into graphics editing software packages and within the shape boundaries create or apply the images which the object will represent. The image layout of the object is then applied to the three dimensional model and from the store vectors the image is calculated and wrapped around the object mesh, creating a realistic model. The common size to use for mapping is image sizes of 256x256, as this size uses less than one megabyte of memory.

\section{IVIDENT Client Architecture}

Figure 2 illustrates the proposed IVIDENT client architecture and consists of a client/server relationship based on a presentation and business layer, the presentation layer consists of a client application and the business layer consists of the server components.

Creating a distributed architecture allows for the development of a thin client application where rendering of graphics and processing system information becomes more efficient due to maintain the visual elements for the user and the business logic layer maintains the system intelligence whereby controlling how the client application will behave, based on how to illustrate the visual elements and delivering effective learning capabilities.

This result in the system becoming easily maintainable and flexible breaking the system into separate components that work together to create an illusion of a single system existing, and further developments made to a layer does not affect the system within other layers and promotes the use of object orientated development.

The client application consists of five layers required to develop a virtual world, graphic engine technologies utilise the graphics card hardware to calculate the gaming physics to enable the rendering of the virtual dental school and dental objects. The client application layer relies on the graphics engine to provide data to display the 
virtual world, and also provides additional functionality to manipulate and customise the virtual dental school.

The game server sends the client application information regards to how the virtual dental school is to be viewed and additional information to update the state of objects present and newly created within the virtual dental school.

Extensible Mark-up Language is used for the configuration settings of the behaviour of the three dimensional models developed, and store information, pointing to where the model is stored, the orientation and scale of the model.

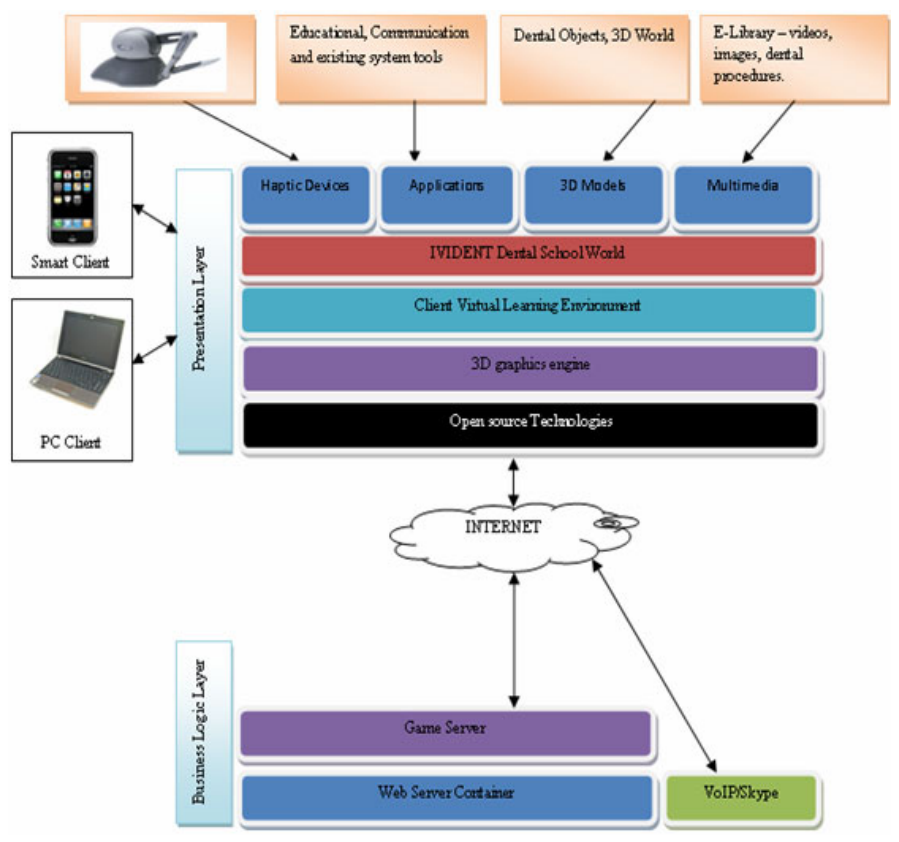

Fig. 2. Virtual Dental School Client/Server Architecture

\section{The Design and Development of the Virtual Dental School}

Figure 3 illustrates the design of the virtual dental school buildings based on a medieval themed virtual world developed using blender three dimensional modelling software package.

Each building represents behaviour of the system based components found within academic institutions, the Keep building defines the library of the virtual world, where end users are able to view and download a large respiratory of multimedia content ranging from e-books to videos which will be integrated into an existing library external system.

The e-commerce market will enable end users to purchase online goods from external online vendors and also trade objects with other users. 


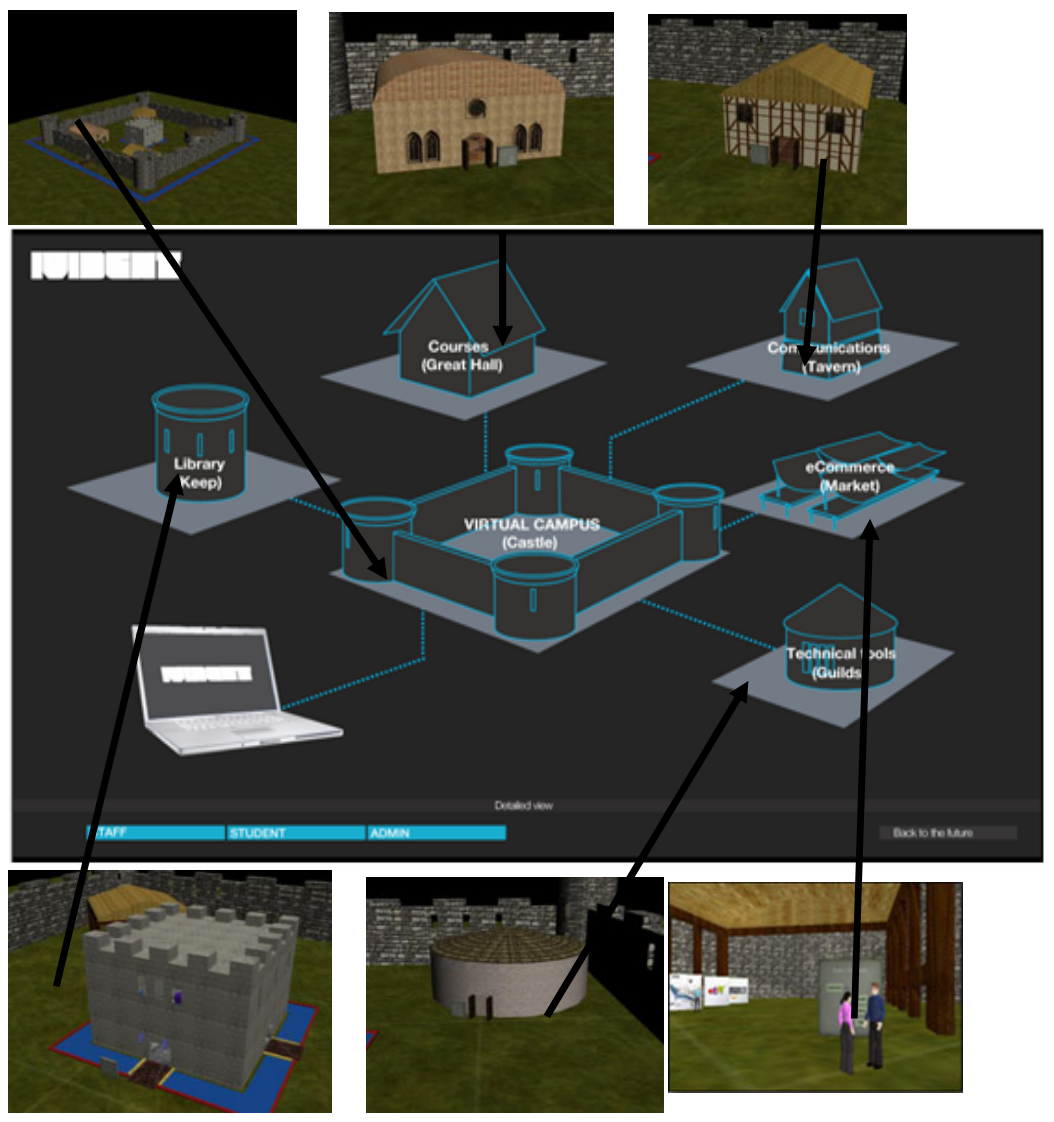

Fig. 3. Virtual Dental School Design

The tavern functionality caters for the social networking aspect of virtual worlds use, where end users are able to collaborate and interact with other end users, via project and group work collaboration, presentations and general text based and video chat tools. An additional functionality of the tavern is the student union.

The guilds building enhances the dental training, by implementing the collaborative ad productive elements of the dental school through rendering dental simulations and objects for end users to interact with and also perform dental procedures. The factor for the guilds is to create an immersive building environment, with detailed realistic dental equipment and models to aid in the engagement in student learning.

The screen shots below illustrate the progress of using Project Wonderland to prove the concepts of using open source technology to develop a flexible online virtual learning environment. The first screenshot illustrates teleportation objects, where the user is able to transport the avatar (human representation of virtual character) to the desired building object which represents an educational component. 

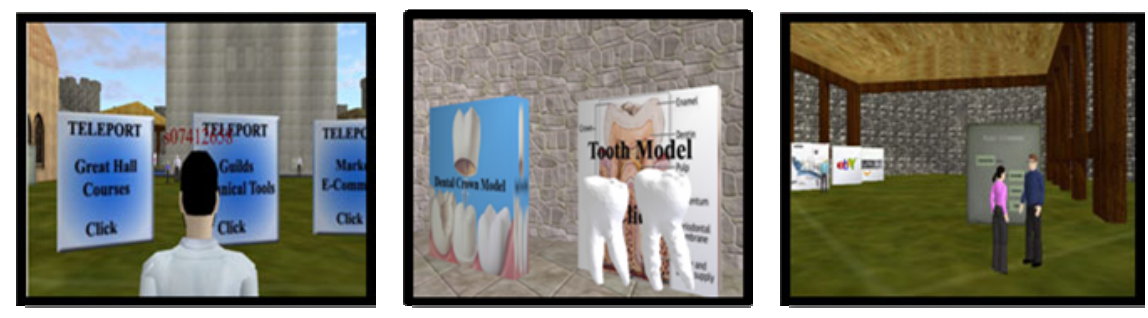

Fig. 4. Virtual dental school development screenshots

The second screenshot illustrates three dimensional teeth models which are rendered dynamically when the end user interacts with the objects providing graphical images of the dental procedure. Using the concept of rendering three dimensional models will allow students to interact and manipulate three dimensional dental objects, to gain further understanding.

The third screenshot illustrates a market area, with the concept of e-commerce, where users of the virtual worlds will be able to purchase online goods.

\section{Conclusion}

Sun Microsystems has release a new version of the virtual world kit project wonderland 0.5 , re-developing the virtual world architecture for flexibility to exist for development of additional project wonderland components. Further development will reside in researching and developing with web technologies and project wonderland 0.5 , the current architecture for project wonderland will be extended via creating an additional data access layer to store persistence of the world in an object oriented database. The client application will also be re-developed, using the existing application as a framework to customise for the requirements of the system, because the current functionality of the client is applicable for general use of virtual worlds and many developers are improving the interface for user needs. This is one of the issues rising with use of project wonderland, the basic framework is provided, but for customisation to suit environment of use, development is inevitable.

Web technologies is also an option for development of client applications, the use of web technologies will allow the system to be accessible on various clients supporting the technology used, allowing students to access the virtual dental school via desktop and smart mobile clients.

Khronos group [7] are developing a new technology webgl which will deliver rich three dimensional content within the browser without the need to install addiotnal plug-ins. The advancement in web graphics technology will create a new interactive experience for end users, and will be researched for developing the visual graphics for IVIDENT virtual dental school.

Haptic technology is advancing, and the next stages will be to develop virtual dental instruments, for students to acquire hands on experience on the realism of performing dental procedures. 


\section{References}

1. Second Life, http: / / secondlife.com/ ?v=1.1 (retrieved January 15, 2010)

2. Marileena Koskela, P.K.: Suitability of a Virtual Learning Environment for Higher Education. Electronic Journal of e-Learning 3(1), 1-30 (2005)

3. Project Life Spans in the 1990s: The Role of the Project Life Cycle (Life Span) in project Management, http: / /www . maxwideman.com/papers/plc-models/1990s.htm (retrived November 16, 2009)

4. Virtual Environments Enable New Models of Learning (n.d.) (2009), http://secondlifegrid.net/slfe/education-use-virtual-world (retrieved November 16, 2009)

5. Project Wonderland: Toolkit for Building 3D Virtual Worlds (2007), Java.net: https : / / lg3d-wonderland.dev.java. net/ (retrieved November 16, 2009)

6. Palmer, I.: Essential Java 3D. Great Britain: The Cromwell Press (2001)

7. Hirshon, J.: Khronos Press Releases from Khronos Group Open Standards for Media authoring and Acceleration(August 4, 2009),

http: / /www . khronos . org/news / press / releases / khronos-webglinitiative-hardware-accelerated-3d-graphics-internet/

(retrieved November 16, 2009) 